1 Smith R, Sykes B. Osteogenesis imperfecta (the brittle bone syndrome) advances and controversies. Calcif Tissue Int 1985;37:107-11.

2 Sykes B. Genetics cracks bone disease. Nature 1987;330:607-8.

3 Stacey A, Bateman J, Choi T, Mascara T, Cole W, Jaenish R. Perinatal lethal osteogenesis imperfecta in transgenic mice bearing an engineered mutant pro-alpha 1 (I) collagen gene. Nature 1988;332:131-6.
4 Holbrook KA, Byers PH. Structural abnormalities in the dorsal collagen and elastic matrix from the skin of patients with inherited connective tissue and elastic matrix from the skin of patients with inherited

5 Bullough PG, Vigorita VJ. Atlas of orthopaedic pathology with clinical and radiographic correlations. London: Butterworths, 1984:3.2-3.8.

6 McAllion SJ, Paterson CR. Causes of death in osteogenesis imperfecta. $f$ Clin Pathol 1996;49:627-630.

\title{
Cytogenetics 40 years on
}

This year marks the 40th anniversary of Tjio and Levan's historic observation that the correct diploid number of human chromosomes is $46 .{ }^{1}$ In common with comparable landmarks, their work represented the culmination of many scientific and technical developments permitting for the first time chromosome preparations of sufficient quality to identify correctly the human chromosome number. Their paper led to the advent of clinical cytogenetics, a genetic discipline which, over the past 40 years, has grown to provide the UK with a comprehensive, regionally based service.

Given the anniversary being celebrated this year, it is appropriate that this issue of the Journal should contain an article presenting the results of a survey which was designed primarily to discover the culture success rates and quality of the cytogenetic service currently being provided by 30 of the UK's cytogenetic laboratories. ${ }^{2}$ As many of the clinicians referring tissue biopsy specimens from spontaneous abortions, fetal remains, stillbirths, and neonatal deaths for chromosome studies are pathologists, the results and recommendations of this study provide useful and practical guidelines on how to optimise the chance of successfully achieving a cytogenetic result from these specimens. One striking result was the high rate of maternal cell contamination following the culture of tissues derived from products of conception, the majority comprising early gestation spontaneous abortions. Although many couples who suffer early pregnancy loss may want to know whether a chromosome abnormality accounted for the miscarriage, the high rate of maternal cell contamination means that, in a high proportion of such specimens, the maternal karyotype is inadvertently examined. The most appropriate test, therefore, in couples with a history of recurrent miscarriages remains the examination of the parental chromosomes.

As cytogeneticists reflect on the first 40 years of their profession, it may be an appropriate time to determine how changes in the provision of cytogenetic services have evolved during this time and how they will develop in the next few years. The basic technical principles used by Tjio and Levan provide the foundation of many of the conventional cytogenetic techniques still widely used. However, cytogenetics has seen many scientific and technical advances over the past 40 years. Of these perhaps the most significant is the use of fluorescence in situ hybridisation (FISH) techniques, which is an approach to genetic diagnosis providing an essential bridge between genes and chromosomes. Most cytogenetic laboratories now routinely offer FISH for the diagnosis of a number of subtle chromosome abnormalities below the resolution of conventional microscopy-for example, microdeletion syndromes including DiGeorge and Williams. ${ }^{3}$ The growth over the past three years in FISH applications has been so rapid that in many centres between 10 and $20 \%$ of all postnatal cytogenetic samples now have one or more FISH tests carried out in order to diagnose or further elucidate a cytogenetic diagnosis.

Like so many innovative techniques, the growth in FISH applications will provide a number of challenges to cytogenetic laboratories throughout the western world. For example, trials are currently under way, most notably in America, to test the efficiency of using FISH with DNA probes in uncultured interphase nuclei as a way of detecting numerical abnormalities without having to examine the chromosomes. This approach, significantly faster than most conventional methods, can only be adopted after careful multicentre trials have been completed and validated but clearly may have a number of advantages, particularly in the field of cytogenetic prenatal diagnosis. ${ }^{4}$ One major disadvantage is that an invasive amniocentesis is still required in order to do the test. The American College of Medical Genetics issued a policy statement in 1993 warning against the overenthusiastic adoption of these new technologies and cautioning that all interphase diagnoses must be confirmed by conventional cytogenetics. ${ }^{5}$

The integration of FISH into cytogenetics not only provides a bridgehead between DNA and the chromosome, but underlines the growing tendency towards an integrated cytogenetic and molecular approach to the diagnosis of an increasing number of genetic conditions. The survey by Rodgers et al was commissioned by the Scientific Committee of the Association of Clinical Cytogeneticists, a professional body founded in 1976, who, in partnership with other professional groups including clinical geneticists, molecular geneticists and genetic nurses, joined forces in January 1996 to form the British Society for Human Genetics (BSHG). ${ }^{6}$ The formation of the BSHG is timely and will provide a much needed forum for increasing the scientific and professional integration of all doctors, nurses, scientists, and technologists currently providing genetic health care services in the UK

Head, Prenatal $\mathcal{G}$ Molecular Cytogenetics,

JOHN A CROLLA

Wessex Regional Genetics Laboratory,

Salisbury Health Care NHS Trust,

Salisbury District Hospital,

Salisbury, Wiltshire SP2 $8 B \mathcal{F}$

1 Tiio JH, Levan A. The chromosome number of man. Heredity 1956;42:1-6. 2 Rodgers CS, Creasy MR, Fitchett M, Maliszewska CT, Pratt NR, Waters J. Solid tissue culture for cytogenetic analysis: a collaborative survey for the Association of Clinical Cytogeneticists. $₹$ Clin Pathol 1996;49:638-641.

3 Adinolf M, Crolla J. Nonisotopic in situ hybridization clinical cytogenetics and gene mapping applications. In: Harris $\mathrm{H}$, Hirschhorn $\mathrm{K}$, eds. Advances in human genetics. New York: Plenum Press, 1994:187-255.

4 Schwartz S. Efficacy and applicability of interphase fluorescence in situ hybridization for prenatal diagnosis. Am $\mathcal{F}$ Hum Genet 1993;52:851-3.

5 American College of Medical Genetics. Prenatal interphase fluorescence in situ hybridization (FISH) policy statement. Am $\mathcal{f}$ Hum Genet 1993;53:526-7.

6 Bobrow M. BSHG is born. 7 Med Genet 1996;33:265. 